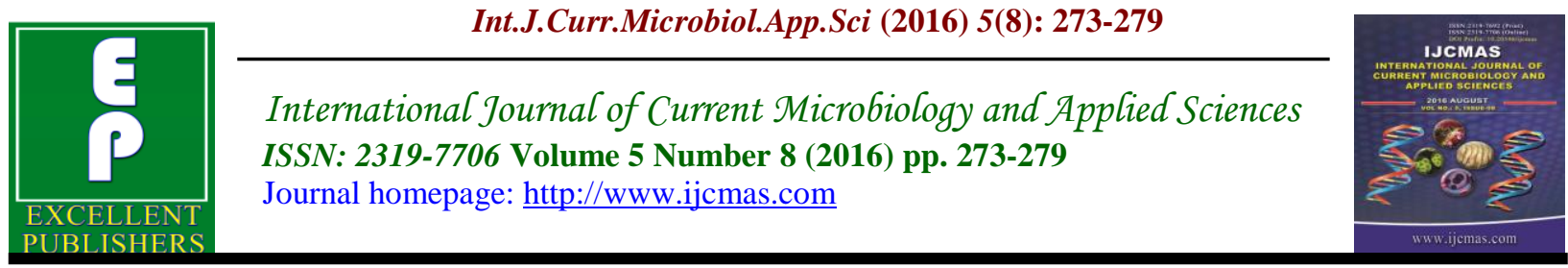

Original Research Article

http://dx.doi.org/10.20546/ijcmas.2016.508.028

\title{
Characterization of Rice Root Transcriptome under Phosphorus Deficiency Stress
}

\author{
Anjali Gupta*, Datta P. Kakade, Jyoti Singh, P.H. Janjal, \\ S.B. Verulkar and Shubha Banerjee
}

Department of Plant Molecular Biology and Biotechnology, College of Agriculture, Indira

Gandhi Krishi Vishwavidyalaya, Raipur - 492001(CG), India

*Corresponding author

\section{A B S T R A C T}

\section{Keywords}

Rice,

Phosphorus,

F2 segregating

lines,

Sahbhagi Dhan X RRF-78.

Article Info

Accepted:

12 July 2016

Available Online:

10 August 2016
Rice (Oryza sativa L.) is the primary source of food, nutrients, energy and employment. Productivity of rice is low usually due to drought salinity, nutrients deficiency etc. Soil phosphorus (P) deficiency is one of the major factors that adversely affecting plant growth causing early maturity in rice and significant reduction in grain yield. Recent studies on mitigation of $\mathrm{P}$ deficiency stress suggests that rice plant exhibits adaptive mechanisms to increase "P" uptake and enhance its utilization under deficient condition and several genes and signaling cascades involved in this response have been reported. As evident from these studies, increase in P uptake alone doesn't provide an advantage over P deficient condition, in terms of higher grain yield which is reflected by $\mathrm{P}$ utilization efficiency (PUE). With this view the present study was conducted to study the response of rice roots in relation to grain yield under $\mathrm{P}$ deficiency stress in $\mathrm{F}_{2}$ segregating lines derived from cross between Sahbhagi Dhan and RRF-78. Field evaluation of the $330 \mathrm{~F}_{2}$ lines indicated significant variations in plant height, total no. of tillers, biological weight and grain yield. For analysis of root trait 5 lines were selected from two extremes. Almost half of all of the 10 genotypes showed increase in root length (RL) and 6 of them showed increase in root volume (RV) and root surface area (RSA). Expression analysis of 9 genes including phosphorus starvation signaling (OsPHR1, OsPHR2, OsPHR3, OsLTN1,OsMYB2P-1), Pi transporter (OsPT1), and NRR locus related genes (Ehd1, Hd3a, RH3) showed differential expression of 5 genes (OsMYB1, OsPHR1, OsPHR2, OsPHR3 and OsPTI) in root tissues under P deficiency stress. On the basis of field evaluation, root trait study and expression analysis, SDXRRF-78(14) and SDXRRF-78(20) were identified as promising $\mathrm{P}$ deficient tolerant genotypes.

\section{Introduction}

Rice (Oryza sativa L.) is the main staple food in Asia, where about $90 \%$ of the world's rice is produced and consumed. It is the primary source of food, nutrients, energy and employment for more than 3.5 billion people across Asia, Africa, and Latin America. Life in Asia depends on rice not 
only because provides $>70 \%$ of the daily calories for the production, but also because of its important role as a source of income for millions of farmers and landless workers (Dawe, 2000). Rice is grown in a variety of environments, covering a wide range of latitudes and altitudes with varying production and productivity as per agroclimatic conditions and soil properties. Productivity of rice is low usually due to soil salinity, nutrients, susceptible varieties to insect pest damages, etc. Soil phosphorus deficiency is one of the major factors adversely affecting rice yield and lack of available phosphorus constrains plant growth in over billion ha of land worldwide (Batjes, 1997; Wissuwa et al., 1998). Phosphorus is an essential macronutrient for normal cell growth and cell division in living organisms also this nutrient plays a vital role in numerous activities such as energy transfer, photosynthesis, nutrient transport, sugar metabolism, and reproduction. Phosphorus $(\mathrm{P})$ is the second most limiting macro nutrient to plant growth and lack of Phosphorus deficiency is one of the most widespread soil problems, against which plants appear to have developed several mechanisms to enhance $\mathrm{P}$ uptake under $\mathrm{P}$ deficient conditions (Raghothama and Karthikeyan, 2005). Low P content in soil may is attributed to low $\mathrm{P}$ content of the parental material, low $\mathrm{pH}$ and/or soil with high P-fixing characteristics (Rose and Wissuwa, 2012). To develop phosphorus tolerance variety can overcome constraints mentioned above. Development of rice varieties with higher phosphorus use efficiency is the major concern for crop biologists and rice growers all over the world. The form of $\mathrm{P}$ most readily accessed by plants is phosphate, the concentration of which rarely exceeds $10 \mathrm{~mm}$ in soil solutions (Bieleski, 1973). So that to utilize available phosphorus, plants must have specialized transporters at the root/soil interface. Studies on P deficiency tolerance in rice have indicated significant genetic variation for $\mathrm{P}$ starvation response adaption of root traits and $\mathrm{P}$ transporter proteins in Oryza gene pool. A few genes such as OsPHR1, OsPHR2, OsPTF1, OsSPX1, OsSPX2, OsSPX3, OsIPS1 and OsIPS2 (Hou et al., 2005; Wang et al., 2009) have been reported in $\mathrm{P}$ deficiency tolerance mechanism, but whether they function similarly in different rice genotypes in response to low $\mathrm{P}$ is not clear. Dai et al., (2012) reported that OsMYB2P-1 involve in regulation of phosphate-starvation responses and activates number of P-responsive genes in rice. An increase in level of expression of high affinity $\mathrm{P}$ transporter has been reported in roots of P-deficient plants.

\section{Materials and Methods}

The present study was conducted at Department of Plant Molecular Biology and Biotechnology and research field under University Instructional field, Indira Gandhi Krishi Vishwavidyalaya, Raipur, (C.G.) The $330 \quad \mathrm{~F}_{2}$ lines (Sahbhagi Dhan X RRF-78) were grown in $\mathrm{P}$ deficient $(\mathrm{P}$ content $7.59 \mathrm{~kg} / \mathrm{ha}$ and $5.87 \mathrm{~kg} / \mathrm{ha} \mathrm{P}_{2} \mathrm{O}_{5}$ in field at 15 $\mathrm{cm}$ and $30 \mathrm{~cm}$ depth respectively) field in kharif 2015. Phenotypic trait such as plant height, no. of tillers/plant, biological yield and grain yield were recorded in phosphorus deficient condition. On the basis of field performance of the rice genotypes, 5 good performing and 5 poor performing lines were selected along with parents (Sahbhagi Dhan and RRF-78). These selected plants were grown for 45 days in rhizotron to characterize the root morphological traits under two conditions i.e. with and without phosphorous supplementation. Observations for plant height, root fresh weight, shoot fresh weight, root dry weight, shoot dry weight, root length, root surface area and root volume were recorded. Root length, 
root surface area and root volume were analyzed with the help of root scanner and WinRhizo software (Fig.2.). For expression analysis of the root sample of genotypes were harvested (45 days after sowing) and frozen in liquid nitrogen for RNA extraction.

RNA was extracted using method with trizol reagent and cDNA synthesis was done using verso cDNA synthesis kit as per manuscript used instructions.

Semi quantitative RT-PCR was performed using 9 genes (OsPHR1, OsPHR2, OsPHR3, OsPT1, OsLTN1, OsMYB2P-1, Ehd1, Hd3a, RH3). The presence of amplicons and their respective intensity were recorded under gel documentation system and relative gene expression levels were compared by the band intensity.

\section{Results and Discussion}

The $330 \mathrm{~F}_{2}$ plants (Sahbhagi Dhan X RRF 78) were evaluated in $P$ deficient field condition, the phenotypic observations under $\mathrm{P}$ deficient condition indicated substantial variation among $\mathrm{F}_{2}$ population of rice genotypes in field. Out of 330 plants only 298 plants produced seeds while 32 plants died or did not produced any seed.

5 plants were selected from two extremes ( 5 good yield and 5 poor yield plants) (Table.1), the selected plants along with parents (Sahbhagi Dhan and RRF-78) for further evaluation in rhizotron to characterize the root morphological traits under two conditions i.e. with and without phosphorous supplementation. A large root system is capable of exploring greater soil volume has therefore been recognized as one important adaptation of plants to ensure a sufficient uptake of P (Sattelmacher et al., 1994; Horst et al., 2001).

Observations for various morphological traits such as plant height, root fresh weight, shoot fresh weight, root dry weight, shoot dry weight, root length, root surface area and root volume. On the basis of rhizotron experiment we have selected SD X RRF-78 (14) and SD X RRF-78 (38) line which showed increase in root surface area and root volume and also found to be high yielding lines.

In the present study observed that SD X RRF-78 (20) is high yielder genotype but their root length, root surface area and root volume decreased in $\mathrm{P}$ deficient condition which indicated role of PUE (Phosphorus use efficiency) marginally in such genotype. Root length, root surface area and root volume increases in SD X RRF-78 (187), SD X RRF-78 (282), SD X RRF-78 (295), SD X RRF-78 (210) lines .Under $P$ deficiency commonly observed in most species that increase in root hair length and density occurs which lead to improve $\mathrm{P}$ uptake through an expansion of root surface area at minimal cost (Gahoonia and Nielsen, 1997; Lynch and Brown, 2001).

In our study 6 out of 10 lines except 4 showed increase in root volume and root surface area (Table.2), which was not necessarily related to grain yield. As only 3 lines (SDXRRF-78 (14), SDXRRF-78 (38), SDXRRF-78 (187)) showed higher grain yield. Which indicated that increase in root volume or surface area doesn't ensure higher grain yield in $\mathrm{p}$ deficiency stress. 
Table.1 Selected genotypes on the basis of field evaluation (5 lines were selected from two extremes)

\begin{tabular}{llcc}
\hline S.No. & Genotypes & Grain Yield (gm) & Bundle wt (gm) \\
\hline $\mathbf{1}$ & Sahbhagi & 11.5 & 30.8 \\
$\mathbf{2}$ & SDXRRF-78(14) & 31 & 141 \\
$\mathbf{3}$ & SDXRRF-78(20) & 30.5 & 172 \\
$\mathbf{4}$ & SDXRRF-78(38) & 36.6 & 133 \\
$\mathbf{5}$ & SDXRRF-78(187) & 35.9 & 159 \\
$\mathbf{6}$ & SDXRRF-78(211) & 51 & 195 \\
$\mathbf{7}$ & SDXRRF-78(199) & 3.7 & 7 \\
$\mathbf{8}$ & SDXRRF-78(210) & 3 & 10 \\
$\mathbf{9}$ & SDXRRF-78(268) & 3.7 & 6 \\
$\mathbf{1 0}$ & SDXRRF-78(282) & 3.6 & 7 \\
$\mathbf{1 1}$ & SDXRRF-78(295) & 3.5 & 7 \\
$\mathbf{1 2}$ & RRF-78 & 12.65 & 33.15 \\
\hline
\end{tabular}

Table.2 Differences in trait value (Root length, Root Surface area and Root Volume) (P0-P1) under P deficient (P0) and P supplement (P1) condition

\begin{tabular}{llll}
\hline Genotypes & Root Length & Root S A & Root Volume \\
Sahbhagi & 0 & 0 & 0 \\
SDXRRF-78(14) & -16.285 & 7.7401 & 0.082 \\
SDXRRF-78(20) & -157.055 & -12.430 & -0.053 \\
SDXRRF-78(38) & -180.495 & 14.1495 & 0.069 \\
SDXRRF-78(187) & 595.8658 & 32.4218 & 0.14 \\
SDXRRF-78(211) & -107.475 & 0 & -0.024 \\
SDXRRF-78(199) & -32.5716 & -3.3587 & -0.025 \\
SDXRRF-78(210) & 61.2063 & 4.797 & 0.027 \\
SDXRRF-78(268) & -105.37 & -6.6102 & -0.036 \\
SDXRRF-78(282) & 450.1602 & 27.9092 & 0.137 \\
SDXRRF-78(295) & 190.2855 & 73.6645 & 0.465 \\
RRF-78 & 0 & 0 & 0 \\
\hline \hline
\end{tabular}

Fig.1 Frequency distribution of (a) Biological wt (gm) and (b) Grain yield (gm) in P deficient field condition in F2 population derived from SD X RRF-78
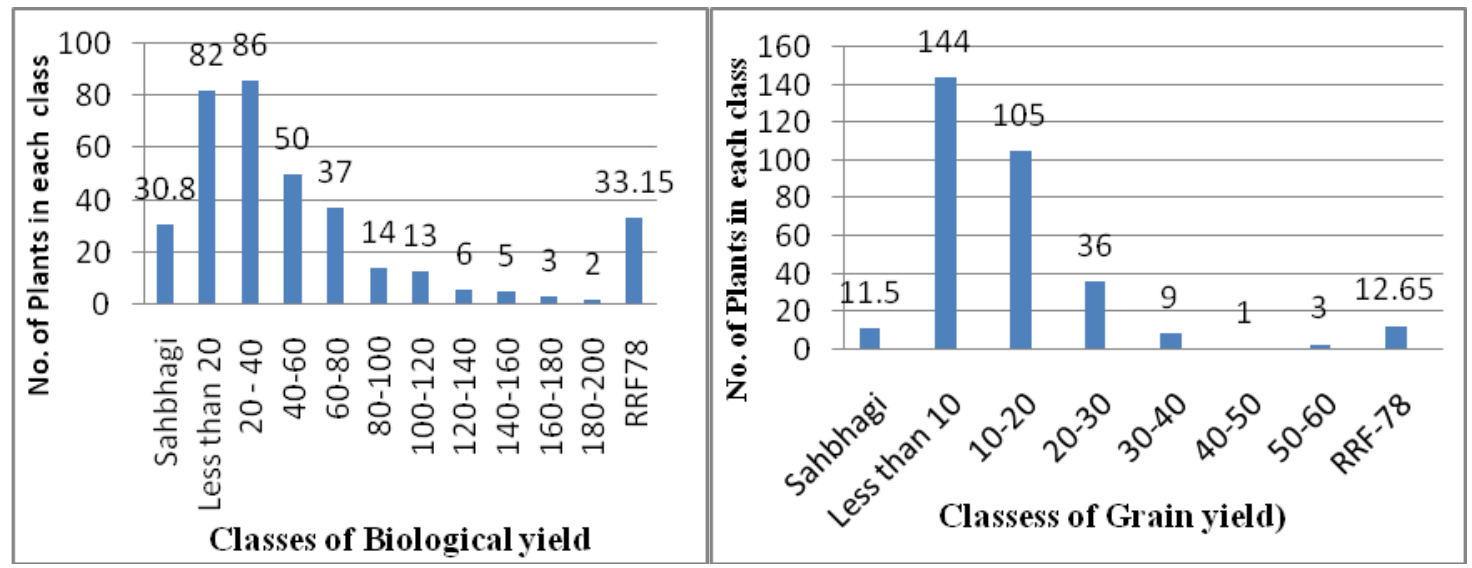
Fig.2 Root length in soil filled rhizotron under $\mathrm{P}$ supplemented condition

$(\mathrm{P}+)$ and $\mathrm{P}$ deficient condition $(\mathrm{P}-)$ (root scanner )

\section{Root scanning}
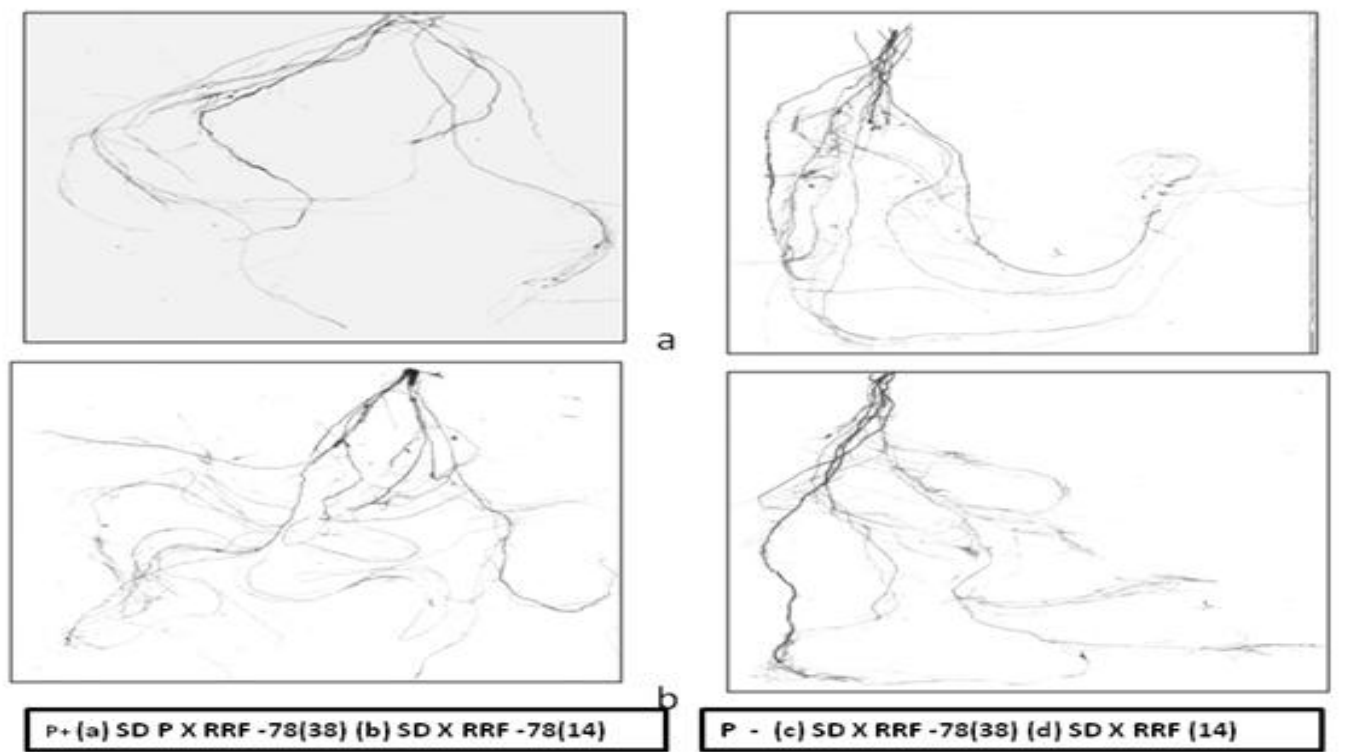

C

P+ (a) SD P X RRF -78(38) (b) SD X RRF -78(14)

P - (c) SD X RRF - 78(38) (d) SD X RRF (14)

Fig.3 Levels of expression of, OsPHR1, OsPHR2, OsPHR3, OsPT1 and OsMYB1 in root of F2 lines (Sahbhagi Dhan and RRF-78 )

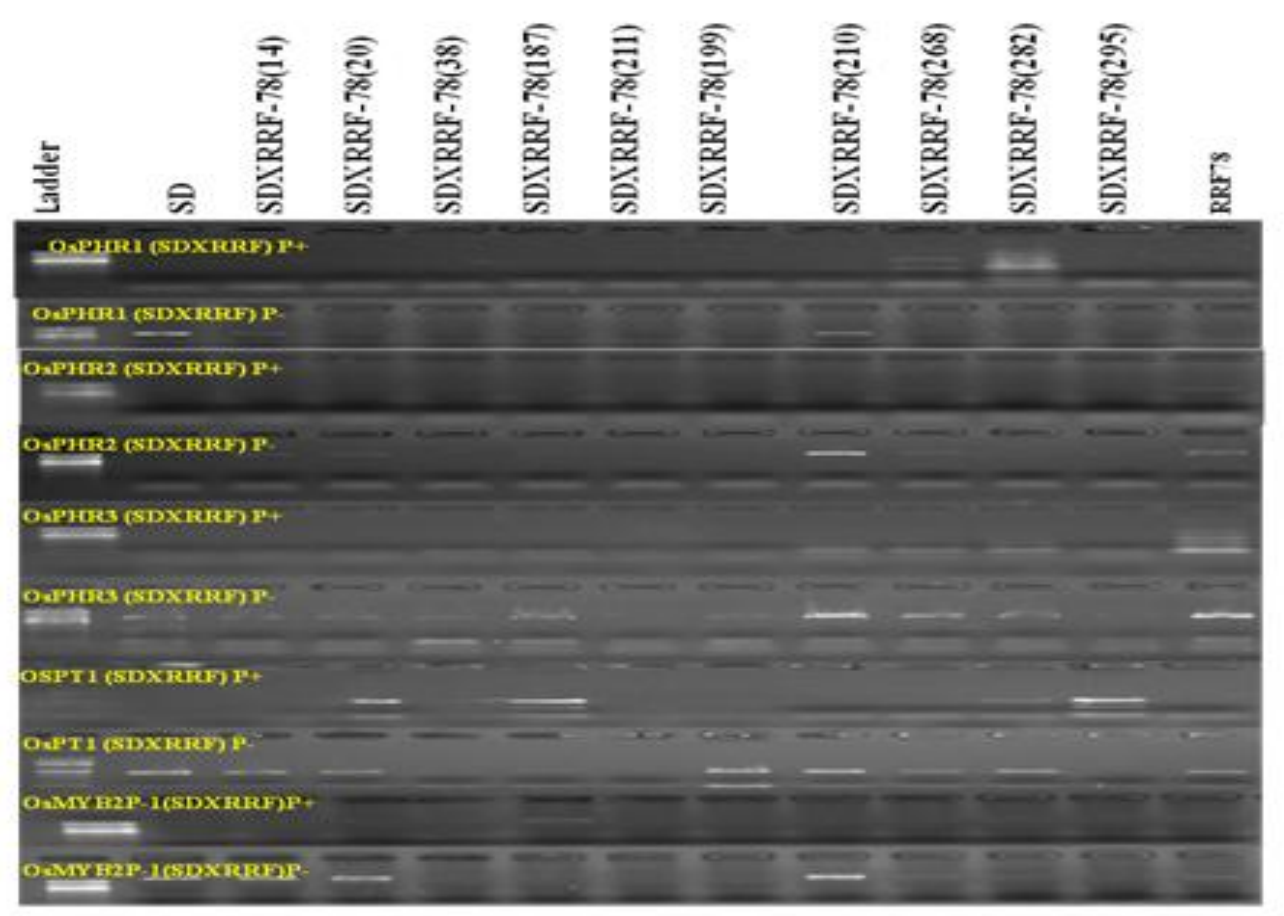

The grain yield is a manifestation of both higher P uptake and also better P utilization effect. PUE has been explained as grain yield per unit of phosphate uptake ((Rose $e t$ 
al., 2011). The PUE of rice depends on ability of plant to sense and signal $P$ deficiency and further to ensure the redistribution of available phosphorus for reproductive growth. Therefore to understand the role of genes related to $\mathrm{P}$ sufficient and deficient related response in rice expression.

Expression analysis of 9 genes including phosphorus starvation signaling (OsPHR1, OsPHR2, OsPHR3, OsLTN1,OsMYB2P-1), $\mathrm{P}$ transporter (OsPT1), ) and NRR locus related genes (Ehd1, Hd3a, RH3) showed differential expression of 5 genes (OsMYB1， OsPHR1，OsPHR2，OsPHR3 and OsPTI) in root tissues under $P$ deficiency stress. Four out of 9 genes did not show any expression in root tissues of neither high yielding nor poor yielding rice lines. Whereas rest of the 5 genes showed differential expression in $\mathrm{P}$ sufficient and $\mathrm{P}$ deficient condition with significant genotypic variations (fig.3.).The expression analysis showed up regulation of all of these 5 genes was recorded in the 2 high yielding genotypes (SD X RRF-14, SD X RRF78 (20) and one susceptible genotype (SD X RRF78 (210). It might be explained by redistribution of available $\mathrm{P}$ for either root or shoot development. As reported by Cheng. el al. 2011, that increase in expression of Pup1 QTL increase the root growth in both tolerant and susceptible genotype. On the basis of field evaluation, root trait study and expression analysis, SDXRRF-78 (14) and SDXRRF-78 (20) were identified as promising $\mathrm{P}$ deficient tolerant genotypes.

\section{Acknowledgement}

I express my heartfelt gratitude to Dr. S.B.Verulkar (HOD, Plant Molecular Biology and Biotechnology), IGKV, Raipur, Chhattisgarh, India for their excellence guidance, suggestions and regular encouragement during the course of investigation.

\section{References}

Batjes, N.H. 1997. A world data set of derived soil properties by FAOUNESCO soil unit for global modeling. Soil Use Manag, 13: 9-16.

Cheng, L., Bucciarelli, B., Liu, J., Zinn, K., Miller, S., Patton-Vogt, J. and Vance, C.P. 2011. White lupin cluster root acclimation to phosphorus deficiency and root hair development involve unique glycerophosphodiester phosphodiesterases. Plant physiol., 156(3): 1131-1148.

Dawe, D. 2000. The potential role of biological nitrogen fixation in meeting future demand for rice and fertilizer. In: Ladha JK, Reddy PM, eds. The request for nitrogen fixation in rice. Philippines: Int. Rice Res. Institute, 1-9.

Gahoonia, T.S., \& Nielsen, N.E. 1997. Variation in root hairs of barley cultivars doubled soil phosphorus uptake. Euphytica, 98(3): 177-182.

Horst, W.J., Kamh, M., Jibrin, J.M., \& Chude, V.O. 2001. Agronomic measures for increasing $\mathrm{P}$ availability to crops. Plant and Soil, 237(2): 211-223.

Lynch, J.P., Brown, K.M. 2001. Topsoil foraging-an architectural adaptation of plants to low phosphorus availability, Plant and Soil,237(2): 225-237.

Raghothama, K.G., Karthikeyan, A.S. 2005. Phosphate acquisition. Plant Soil, 274:37-49.

Rose, T.J., Rose, M.T., Pariasca Tanaka, J., Heuer, S., \& Wissuwa, M. 2011. The frustration with utilization: why have improvements in internal phosphorus utilization efficiency in crops 
remained so elusive? Frontiers in Plant Sci., 2: 73.

Rose, T.J., \& Wissuwa, M. 2012. Rethinking internal phosphorus utilization efficiency: a new approach is needed to improve PUE in grain crops. Adv. Agronomy, 116: 185-217.

Sattelmacher, B., Horst, W.J., \& Becker, H.C. 1994. Factors that contribute to genetic variation for nutrient efficiency of crop plants. Zeitschrift für Pflanzenernährung und Bodenkunde, 157(3): 215-224.

USDA Annual report on Food \& Nutrition 2012.

Wissuwa, M., Yano, M. and Ae, N. 1998. Mapping of QTLs for phosphorus deficiency tolerance in rice (Oryza sativa L.). Theor. Appl. Genet., 97: $777-783$.

\section{How to cite this article:}

Anjali Gupta, Datta P. Kakade, Jyoti Singh, P.H. Janjal, S.B. Verulkar and Shubha Banerjee. 2016. Characterization of Rice Root Transcriptome under Phosphorus Deficiency Stress. Int.J.Curr.Microbiol.App.Sci. 5(8): 273-279. doi: http://dx.doi.org/10.20546/ijcmas.2016.508.028 